PROCEEDINGS OF THE

AMERICAN MATHEMATICAL SOCIETY

Volume 116, Number 3, November 1992

\title{
CONTINUOUS DENSE EMBEDDINGS OF STRONG MOORE ALGEBRAS
}

\author{
MICHAEL J. MEYER
}

(Communicated by Palle E. T. Jorgensen)

\begin{abstract}
We introduce the class of strong Moore Banach algebras (all topologically transitive representations of a certain type are finite dimensional) and investigate stability properties with respect to completions of such algebras in continuous submultiplicative norms. Among other things it is shown that every irreducible representation of a regular (definition below) strong Moore Banach algebra $\mathscr{A}$ extends to all Banach algebras in which $\mathscr{A}$ is continuously and densely embedded.
\end{abstract}

\section{INTRODUCTION AND RESULTS}

One of the earliest results about submultiplicative norms on Banach algebras is the following fact established by I. Kaplansky in [7]: If $\mathscr{A}$ is the algebra $\mathscr{C}(X)$ of all continuous functions on a locally compact Hausdorff space $X$ that vanish at infinity endowed with the familiar supremum norm $|f|_{\infty}$, then every submultiplicative norm \|\| on $\mathscr{A}$ satisfies

$$
\|f\| \geq|f|_{\infty} \text { for all } f \in \mathscr{A} .
$$

This result was the beginning of a series of deep investigations into the nature of norms on $\mathscr{C}(X)$ [8]. In the above form (0) cannot be extended to any semisimple noncommutative algebra. In fact, if all (submultiplicative) norms on a Banach algebra $\mathscr{A}$ dominate the complete norm || of $\mathscr{A}$ as in (0), it follows from [12, I.4.2] that $|x|=\rho_{\mathscr{A}}(x)$ for all $x \in \mathscr{A}$, where $\rho_{\mathscr{A}}$ denotes the spectral radius on $\mathscr{A}$. In particular, $\rho_{\mathscr{A}}$ is subadditive on $\mathscr{A}$ and hence $\mathscr{A}$ is commutative [9, Theorem 2.1.2]. On the other hand the proof of (0) relies on the fact that for the algebra $\mathscr{C}(X)$ the complete norm is the spectral radius, so that $(0)$ should be written more properly as

$$
\|a\| \geq \rho_{\mathscr{A}}(a) \text { for all } a \in \mathscr{A},
$$

in this form, (1) is valid (for all norms on $\mathscr{A}$ ) for several important classes of noncommutative algebras [10].

Norms satisfying (1) are called $Q$-norms on $\mathscr{A}$ and share many desirable properties with complete norms. For example, the group of invertibles of a

Received by the editors April 1, 1991.

1991 Mathematics Subject Classification. Primary 46H10, 46H15.

Key words and phrases. Extension of finite dimensional representations. 
unital Banach algebra is open in every $Q$-norm, and any two $Q$-norms \|\|$_{1},\|\|_{2}$ on a semisimple Banach algebra $\mathscr{A}$ are consistent in the sense that the identity map $\left(\mathscr{A},\|\|_{1}\right) \rightarrow\left(\mathscr{A},\|\|_{2}\right)$ has closed graph. For a full survey see $[10,11]$.

In several situations in analysis inequality (1) has significance even if only a single norm is considered. For example, assume that $\mathscr{A}$ is a ${ }^{*}$-semisimple Banach algebra with involution. Then $\mathscr{A}$ is symmetric if and only if the largest $C^{*}$-norm on $\mathscr{A}$ is a $Q$-norm. Furthermore, any two $C^{*}$-norms on $\mathscr{A}$ that satisfy (1) are equal. Therefore, if all norms on $\mathscr{A}$ are $Q$-norms, then $\mathscr{A}$ carries a unique $C^{*}$-norm, a useful property with various consequences [4]. If $\mathscr{A}$ is the group algebra $L^{1}(G)$ of a locally compact group $G$, then $G$ is amenable and symmetric if and only if the $C^{*}$-norm induced by the left regular representation of $\mathscr{A}$ on $L^{2}(G)$ is a $Q$-norm. All the norms mentioned here are continuous. These examples show that it is interesting to know under which conditions every norm, or at least every continuous norm, on $\mathscr{A}$ is a $Q$-norm, not merely because this is an extension of $(0)$.

Suppose now that \|\| is a norm on $\mathscr{A}$, and let $\mathscr{B}$ be the completion of $\mathscr{A}$ in this norm. It is easy to see that (1) holds if and only if $\rho_{\mathscr{B}}(a)=\rho_{\mathscr{A}}(a)$, for all $a \in \mathscr{A}$, and this follows from $\operatorname{Sp}_{\mathscr{A}}(a) \cup\{0\} \subseteq \mathrm{Sp}_{\mathscr{B}}(a) \cup\{0\}$, for all $a \in \mathscr{A}$. One always has $\operatorname{Sp}_{\mathscr{B}}(a) \cup\{0\} \subseteq \operatorname{Sp}_{\mathscr{A}}(a) \cup\{0\}$. In the class of algebras $\mathscr{A}$, which we will study below, for every $\lambda \in \operatorname{Sp}_{\mathscr{A}}(a), \lambda \neq 0$, there exists an irreducible representation $\pi$ of $\mathscr{A}$ on some (finite-dimensional) Banach space $X$ such that $\lambda \in \operatorname{Sp}(\pi(a))$, where this latter spectrum is computed in $\mathscr{B}(X)$. If the representation $\pi$ extends to $\mathscr{B}$ it will follow that $\lambda \in \operatorname{Sp}_{\mathscr{B}}(a)$. This naturally leads to the question: under which circumstances do irreducible representations of $\mathscr{A}$ extend to algebras $\mathscr{B}$ in which $\mathscr{A}$ is densely embedded?

The first result about the possibility of extensions was given by Shilov in [1]: Without assumptions on the commutative Banach algebra $\mathscr{A}$, every multiplicative linear functional in the Shilov boundary of $\mathscr{A}$ extends to all Banach algebras $\mathscr{B}$ in which $\mathscr{A}$ is isometrically embedded. Later Rickart showed in [2]: If $\mathscr{A}$ is a semisimple, completely regular, commutative Banach algebra then every multiplicative linear functional on $\mathscr{A}$ extends to all Banach algebras in which $\mathscr{A}$ is (not necessarily continuously) embedded.

Notice that such extension is not always possible even if the embedding is continuous and has dense range. The Stone Weierstrass theorem shows that the disc algebra $\mathscr{A}(D)$ is continuously and densely embedded in the algebra $\mathscr{C}([0,1])$ of all continuous functions on the unit interval. No evaluation functional $f \rightarrow f(z)$, where $z \in D \backslash[0,1]$, extends from $\mathscr{A}(D)$ to $\mathscr{C}([0,1])$.

Below we define the class of strong Moore Banach algebras, which is sufficiently close to the commutative case so as to allow a generalization of Rickart's result in a weakened form (continuity and density of the embedding are assumed). It is shown that this class of Banach algebras has pleasant stability properties with respect to continuous dense embeddings.

Let $\mathscr{A}$ be a Banach algebra. Recall $[3,2.2]$ that a representation of $\mathscr{A}$ on a vector space $X$ is a homomorphism $\pi$ of $\mathscr{A}$ into the algebra $L(X)$ of all linear operators on $X$. The representation $\pi$ is called irreducible if there is no nonzero proper subspace of $X$ that is invariant for all the operators $\pi(a)$, $a \in \mathscr{A}$. It is known that then $\pi$ is transitive on $X$ : for any linearly independent set of vectors $\left\{x_{1}, x_{2}, \ldots, x_{n}\right\} \subseteq X$ and any vectors $y_{1}, y_{2}, \ldots, y_{n} \in X$ there exists $a \in \mathscr{A}$ with $\pi(a) x_{j}=y_{j}$ for all $j=1, \ldots, n$. The representation 
$\pi$ of $\mathscr{A}$ on $X$ is called finite dimensional if $X$ is finite dimensional. If $\pi$ is finite dimensional and irreducible and hence transitive, it follows that $\pi(\mathscr{A})=\mathscr{B}(X)$.

Suppose now that $X$ is a normed space. Then the representation $\pi$ of $\mathscr{A}$ on $X$ is called continuous if all the operators $\pi(a), a \in \mathscr{A}$, on $X$ are continuous and $\pi$ is continuous as a homomorphism of $\mathscr{A}$ into the algebra $\mathscr{B}(X)$ of all bounded linear operators on $X, \pi$ is called topologically irreducible, if there is no nonzero proper closed subspace of $X$ that is invariant under all the operators $\pi(a), a \in \mathscr{A}$, and topologically transitive if for $\left\{x_{1}, x_{2}, \ldots, x_{n}\right\} \subseteq X$ and $y_{1}, y_{2}, \ldots, y_{n} \in X$ as above, there exists a sequence $\left(a_{n}\right) \subseteq \mathscr{A}$ such that $\pi\left(a_{n}\right) x_{j} \rightarrow y_{j}$ as $n \rightarrow \infty$ for all $j=1, \ldots, n$.

Suppose now that $\mathscr{B}$ is another Banach algebra and $\phi: \mathscr{A} \rightarrow \mathscr{B}$ is a continuous homomorphism with dense range. If $\pi: \mathscr{B} \rightarrow \mathscr{B}(X)$ is a continuous irreducible representation of $\mathscr{B}$ on the normed space $X$, then $\pi \circ \phi$ is a continuous topologically transitive representation of $\mathscr{A}$ on $X$. A representation of $\mathscr{A}$ of this form will be called a standard continuous topologically transitive representation of $\mathscr{A}$.

Let us call $\mathscr{A}$ a Moore algebra, if all irreducible representations of $\mathscr{A}$ are finite dimensional, and a strong Moore algebra, if all standard continuous topologically transitive representations of $\mathscr{A}$ are finite dimensional. Our terminology is chosen in honour of Calvin C. Moore who characterized the locally compact groups all of whose irreducible ${ }^{*}$-representations are finite dimensional [13].

If $\pi$ is an irreducible representation of $\mathscr{A}$ on the vector space $X$ then [3, 2.2.6] $X$ can be endowed with a complete vector space norm for which the representation $\pi$ becomes continuous. This shows that every irreducible representation of $\mathscr{A}$ is a standard continuous topologically transitive representation of $\mathscr{A}$. It follows that every strong Moore algebra is a Moore algebra. The following proposition furnishes examples of strong Moore algebras. Recall that a Moore group is a locally compact group $G$ such that all its irreducible strongly continuous ${ }^{*}$-representations on Hilbert space are finite dimensional.

Proposition 1. (A) Every commutative Banach algebra is a strong Moore algebra.

(B) If $G$ is a Moore group then the Banach algebra $L^{1}(G)$ is a strong Moore algebra.

Proof. (A) Let $\mathscr{A}$ be a commutative Banach algebra, $\phi: \mathscr{A} \rightarrow \mathscr{B}$ a homomorphism of $\mathscr{A}$ into the Banach algebra $\mathscr{B}$ with dense range and $\pi$ an irreducible representation of $\mathscr{B}$. The subalgebra $\phi(\mathscr{A}) \subseteq \mathscr{B}$ is commutative and dense in $\mathscr{B}$. It follows that $\mathscr{B}$ is commutative. But then $\pi$ is one dimensional.

(B) If $G$ is a Moore group then $[4,5]$ every continuous topologically irreducible representation of $L^{1}(G)$ on a normed space is finite dimensional.

Remark. It is known that there exist (necessarily infinite-dimensional) Banach spaces $X$ and bounded linear operators $T$ on $X$ that do not have a nontrivial closed invariant subspace. Then the closed subalgebra generated by $T$ in $\mathscr{B}(X)$ is clearly commutative and has a topologically irreducible representation on the infinite-dimensional Banach space $X$. It is for this reason that we restrict ourselves to standard representations.

Let $\rho$ denote the spectral radius on the Banach algebra under consideration. For $a \in \mathscr{A}$ define the permanent radius $\rho_{p}(a)$ by $\rho_{p}(a)=\inf _{\phi} \rho(\phi(a))$, where 
the infimum is taken over all continuous embeddings of $\mathscr{A}$ into another Banach algebra. If $\phi$ is a continuous embedding of $\mathscr{A}$ into another Banach algebra, then $\rho_{p}(\phi(a)) \geq \rho_{p}(a)$ for all $a \in \mathscr{A}$.

Let $\operatorname{Prim}(\mathscr{A})$ denote the primitive ideal space of $\mathscr{A}$ endowed with the hull kernel topology (Jacobson topology). Suppose that $\mathscr{A}$ is a Moore algebra and let $P \in \operatorname{Prim}(\mathscr{A})$. Choose a (necessarily finite-dimensional) irreducible representation $\pi: \mathscr{A} \rightarrow \mathscr{B}(X)$ such that $P=\operatorname{ker}(\pi)$. Then $\pi(\mathscr{A})=\mathscr{B}(X)$ and thus $\mathscr{A} / P \cong \mathscr{B}(X)$, which latter algebra is simple with identity. It follows that the ideal $P \subseteq \mathscr{A}$ is maximal with modular identity. Thus for a Moore algebra $\mathscr{A}$ the primitive ideal space $\operatorname{Prim}(\mathscr{A})$ coincides with the space of modular maximal ideals of $\mathscr{A}$.

Call the Banach algebra $\mathscr{A}$ regular if the kernel of each proper compact subset $F \subseteq \operatorname{Prim}(\mathscr{A})$ contains an element $a$ with $\rho_{p}(a)>0$. Examples of elements of positive permanent radius are furnished by the following

Proposition 2 [6]. Let $\mathscr{A}$ be a Banach algebra.

(1) If $a \in \mathscr{A}$ is a quasi-divisor of zero ( $a x=x$ or $x a=x$ for some nonzero $x \in \mathscr{A})$ then $\rho_{p}(a) \geq 1$.

(2) If $a, h \in \mathscr{A}$ are such that $a h=h a$ is a quasi-divisor of zero then $\rho_{p}(a) \geq 1 / \rho_{\mathscr{A}}(h)$.

(3) $\quad \rho_{p}(a) \geq \operatorname{dist}(C, 0)$, where $C$ is any connected component of $\operatorname{Sp}_{\mathscr{A}}(a)$.

Recall $[3,2.7]$ that $\mathscr{A}$ is called completely regular if the space of modular maximal ideals of $\mathscr{A}$ is Hausdorff and every point $P$ has a neighbourhood $V$ such that the kernel $\operatorname{ker}(V) \subseteq \mathscr{A}$ is modular. Our main results can now be stated as follows:

Theorem 1. Let $\mathscr{A}$ be a strong Moore algebra, $\mathscr{B}$ any Banach algebra, and $\phi: \mathscr{A} \rightarrow \mathscr{B}$ a continuous homomorphism with dense range. Then

(A) $\mathscr{B}$ is a strong Moore algebra.

(B) For each primitive ideal $Q \subseteq \mathscr{B}, P=\phi^{-1}(Q) \subseteq \mathscr{A}$ is a primitive ideal in $\mathscr{A}$ and the map $Q \in \operatorname{Prim}(\mathscr{B}) \rightarrow P=\phi^{-1}(Q) \in \operatorname{Prim}(\mathscr{A})$ is continuous and one to one.

(C) If $\mathscr{A}$ is semisimple and completely regular then $\mathscr{A}$ is regular.

(D) If $\mathscr{A}$ is completely regular then $\mathscr{B}$ is completely regular.

(E) If $\mathscr{A}$ is regular and $\phi$ is an embedding then $\mathscr{B}$ is regular.

Theorem 2. Now let $\mathscr{A}$ be a unital, semisimple, regular, strong Moore algebra, $\mathscr{B}$ any Banach algebra, and $\phi: \mathscr{A} \rightarrow \mathscr{B}$ a continuous embedding with dense range. Then
(A) The map $Q \in \operatorname{Prim}(\mathscr{B}) \rightarrow P=\phi^{-1}(Q) \in \operatorname{Prim}(\mathscr{A})$ is surjective. If $\mathscr{A}$ is completely regular then it is a homeomorphism.
(B) Every irreducible representation $\pi$ of $\mathscr{A}$ extends to a (necessarily irre- ducible) representation of $\mathscr{B}$.
(C) $\operatorname{rad}(\mathscr{B}) \cap \mathscr{A}=(0)$.
(D) The subalgebra $\phi(\mathscr{A}) \subseteq \mathscr{B}$ is inverse closed in $\mathscr{B}: a \in \mathscr{A}, \phi(a) \in$ $\operatorname{inv}(\mathscr{B}) \Rightarrow a \in \operatorname{inv}(\mathscr{A})$.
(E) For all elements $a \in \mathscr{A}$ we have $\operatorname{Sp}_{\mathscr{B}}(\phi(a))=\operatorname{Sp}_{\mathscr{G}}(a)$ and $\rho_{\mathscr{B}}(\phi(a))=$ $\rho_{\mathscr{A}}(a)$. 
(F) Every continuous (not necessarily complete) submultiplicative norm on $\mathscr{A}$ satisfies

$$
\rho_{\mathscr{A}}(a)=\lim _{n \uparrow \infty}\left\|a^{n}\right\|^{1 / n} \leq\|a\| \text { for all } a \in \mathscr{A} .
$$

Theorem 3. Let $\mathscr{A}$ be a semisimple, completely regular, strong Moore algebra (not necessarily unital) and $\mathscr{B}, \phi$ as in Theorem 2. Then all the conclusions of Theorem 2 except (D) still hold. (E) has to be replaced by $\operatorname{Sp}_{\mathscr{B}}(\phi(a)) \cup\{0\}=$ $\mathrm{Sp}_{\mathscr{A}}(a) \cup\{0\}$ and $\rho_{\mathscr{B}}(\phi(a))=\rho_{\mathscr{A}}(a)$ for all $a \in \mathscr{A}$.

\section{Proofs}

Lemma 1. Let $\mathscr{A}, \mathscr{B}$ be Banach algebras and $\phi: \mathscr{A} \rightarrow \mathscr{B}$ a continuous homomorphism with dense range.

(A) If $\pi$ is a standard continuous topologically transitive representation of $\mathscr{B}$, then $\pi \circ \phi$ is a like representation of $\mathscr{A}$.

(B) If $\mathscr{A}$ is a strong Moore algebra then so is $\mathscr{B}$.

Proof. Clearly (B) follows from (A), which is true since a composition of continuous homomorphisms with dense ranges is another such homomorphism.

Lemma 2. Let $\mathscr{A}$ be a strong Moore algebra, $\mathscr{B}$ a Banach algebra and $\phi: \mathscr{A} \rightarrow$ $\mathscr{B}$ a continuous homomorphism with dense range. Then

(A) Every standard continuous topologically transitive representation $\pi$ of $\mathscr{A}$ is in fact (algebraically) transitive.

(B) For each primitive ideal $Q \subseteq \mathscr{B}, P=\phi^{-1}(Q)$ is a primitive ideal in $\mathscr{A}$ and the natural map $a+P \in \mathscr{A} / P \rightarrow \phi(a)+Q \in \mathscr{B} / Q$ is an isomorphism.

Proof. (A) Simply because $\pi$ is topologically irreducible and finite dimensional, hence (algebraically) irreducible.

(B) Let $Q \in \operatorname{Prim}(\mathscr{B})$. Choose a continuous irreducible representation $\pi$ of $\mathscr{B}$ on some Banach space $X$ with $Q=\operatorname{ker}(\pi)$. Then $P=\phi^{-1}(Q)=\operatorname{ker}(\pi \circ \phi)$. Also $\pi \circ \phi$ is a standard continuous topologically transitive representation of $\mathscr{A}$ on $X$, hence finite dimensional and (algebraically) transitive. Then, since $X$ is finite dimensional, $\pi(\phi(\mathscr{A}))=\mathscr{B}(X)=\pi(\mathscr{B})$. Thus $\mathscr{A} / P \cong \mathscr{B}(X) \cong$ $\mathscr{B} / Q$, in particular $\mathscr{A} / P, \mathscr{B} / Q$ are finite-dimensional algebras of the same dimension. The natural map $a+P \in \mathscr{A} / P \rightarrow \phi(a)+Q \in \mathscr{B} / Q$, which is clearly an embedding, therefore, must be onto for reasons of dimension.

Lemma 3. Let $\pi: \mathscr{A} \rightarrow \mathscr{B}(X), \sigma: \mathscr{A} \rightarrow \mathscr{B}(Y)$ be irreducible representations of the Banach algebra $\mathscr{A}$ on finite-dimensional Banach spaces $X, Y$. If $\operatorname{ker}(\pi)=$ $\operatorname{ker}(\sigma)$ then there exists a linear isomorphism $U: Y \rightarrow X$ such that $\sigma(a)=$ $U^{-1} \pi(a) U$ for all $a \in \mathscr{A}$.

Proof. Let $P=\operatorname{ker}(\pi)=\operatorname{ker}(\sigma)$. Then the homomorphisms $\pi, \sigma$ induce isomorphisms $\pi_{0}: \mathscr{A} / P \rightarrow \mathscr{B}(X)$ and $\sigma_{0}: \mathscr{A} / P \rightarrow \mathscr{B}(Y)$. In particular, $\sigma_{0} \pi_{0}^{-1}: \mathscr{B}(X) \rightarrow \mathscr{B}(Y)$ is an isomorphism. Since $X, Y$ are finite dimensional, it is well known that there exists a linear isomorphism $U: Y \rightarrow X$ such that $\sigma_{0} \pi_{0}^{-1}(T)=U^{-1} T U$ for all $T \in \mathscr{B}(X)$. Now let $T=\pi(a)$ to conclude that $\sigma(a)=U^{-1} \pi(a) U$ for all $a \in \mathscr{A}$. 
Proof of Theorem 1. (A) This is Lemma 1(B).

(B) Let $Q \in \operatorname{Prim}(\mathscr{B})$ and choose a continuous irreducible representation $\pi$ of $\mathscr{B}$ with $Q=\operatorname{ker}(\pi)$. Then $\pi \circ \phi$ is a standard continuous topologically transitive representation of $\mathscr{A}$ and thus, by Lemma $2(\mathrm{~A})$, an irreducible representation of $\mathscr{A}$. It follows that $\phi^{-1}(Q)=\operatorname{ker}(\pi \circ \phi) \in \operatorname{Prim}(\mathscr{A})$. Since the inverse images of hulls are hulls, the map $Q \in \operatorname{Prim}(\mathscr{B}) \rightarrow \phi^{-1}(Q) \in$ $\operatorname{Prim}(\mathscr{A})$ is continuous. It remains to be shown that it is one to one. Suppose that $N, Q \in \operatorname{Prim}(\mathscr{B})$ are such that $\phi^{-1}(N)=\phi^{-1}(Q)$. Choose continuous irreducible representations $\pi, \sigma$ of $\mathscr{B}$ on Banach spaces $X, Y$ such that $N=\operatorname{ker}(\pi)$ and $Q=\operatorname{ker}(\sigma)$. Since $\mathscr{B}$ is again a strong Moore algebra and hence a Moore algebra, $X, Y$ are finite dimensional. Furthermore $\operatorname{ker}(\pi \circ \phi)=\operatorname{ker}(\sigma \circ \phi)$ and $\pi \circ \phi, \sigma \circ \phi$ are (algebraically) irreducible representations of $\mathscr{A}$ on $X, Y$. By Lemma 3 there exists a linear isomorphism $U: Y \rightarrow X$ such that $\sigma(b)=U^{-1} \pi(b) U$ for all $b \in \phi(\mathscr{A})$. Now density of $\phi(\mathscr{A})$ in $\mathscr{B}$ implies that this relation holds for all $b \in \mathscr{B}$. It follows that $N=\operatorname{ker}(\pi)=\operatorname{ker}(\sigma)=Q$.

(C) Assume that $\mathscr{A}$ is semisimple and completely regular. Recall that the primitive ideal space $\operatorname{Prim}(\mathscr{A})$ coincides with the space of modular maximal ideals of $\mathscr{A}$, since $\mathscr{A}$ is a Moore algebra, and, therefore, is locally compact [2, 2.7]. Now let $K \subseteq \operatorname{Prim}(\mathscr{A})$ be a proper compact subset. By local compactness we can choose nonempty open subsets $U, V \subseteq \operatorname{Prim}(\mathscr{A})$ such that $\bar{U} \subseteq V \subseteq$ $\bar{V} \subseteq \operatorname{Prim}(\mathscr{A}) \backslash K$. Set $I=\operatorname{ker}(K), F=\operatorname{Prim}(\mathscr{A}) \backslash V$, and $J=\operatorname{ker}(F)$. Then $K, F$ are closed, that is, $K=\operatorname{hull}(I)$ and $F=\operatorname{hull}(J)$. Thus $\bar{V} \cap \operatorname{hull}(I)=$ $\bar{V} \cap K=\varnothing$. By [2, Corollary 2.7.11], $I$ contains an identity $e$ for $\mathscr{A}$ modulo $\operatorname{ker}(\bar{V})$. Then $e \in I=\operatorname{ker}(K)$ and $e+P$ is an identity for $\mathscr{A} / P$ for every $P \in \bar{V}$. Similarly, $\bar{U} \cap$ hull $(J)=\bar{U} \cap F=\varnothing$ and thus $J$ contains an identity $x$ for $\mathscr{A}$ modulo $\operatorname{ker}(\bar{U})$. In particular then $x \in J=\operatorname{ker}(F)$ and $x \neq 0$. Now let $P \in \operatorname{Prim}(\mathscr{A})$ be any primitive ideal. Then either $P \in V \subseteq \bar{V}$, in which case $e x-x \in P$ since $e$ is an identity for $\mathscr{A}$ modulo $P$, or $P \in F$ in which case $e x-x \in P$ since $x \in P$. Thus $e x-x \in \cap \operatorname{Prim}(\mathscr{A})=\operatorname{rad}(\mathscr{A})=(0)$, that is, $e x=x$. Now $x \neq 0$ implies $\rho_{p}(e) \geq 1$.

(D) Since a Banach algebra $\mathscr{A}$ is completely regular if and only if its unitization is completely regular, we may assume that $\mathscr{A}, \mathscr{B}$ are both unital. Then complete regularity of $\mathscr{A}, \mathscr{B}$ is equivalent to the Hausdorff property of the space of modular maximal ideals that coincides with the primitive ideal space of $\mathscr{A}$ and $\mathscr{B}$. By $(B)$ there exists a continuous, injective map $\operatorname{Prim}(\mathscr{B}) \rightarrow$ $\operatorname{Prim}(\mathscr{A})$. Thus the Hausdorff property of $\operatorname{Prim}(\mathscr{A})$ implies the Hausdorff property for $\operatorname{Prim}(\mathscr{B})$.

(E) Assume that $\mathscr{A}$ is regular and $\phi$ one to one. Then $\rho_{p}\left(\phi(a) \geq \rho_{p}(a)\right.$ for all $a \in \mathscr{A}$. Now let $K \subseteq \operatorname{Prim}(\mathscr{B})$ be a proper compact subset. According to (B) the map $\Phi: Q \in \operatorname{Prim}(\mathscr{B}) \rightarrow \phi^{-1}(Q) \in \operatorname{Prim}(\mathscr{A})$ is continuous and one to one. It follows that $\Phi(K)$ is a proper compact subset of $\operatorname{Prim}(\mathscr{A})$. Since $\mathscr{A}$ is regular, there exists an element $a \in \operatorname{ker}(\Phi(K))$ such that $\rho_{p}(a)>0$. But then $\phi(a) \in \operatorname{ker}(K)$ and $\rho_{p}(\phi(a)>0$.

Proof of Theorem 2. We may assume that $\mathscr{A} \subseteq \mathscr{B}$ and $\phi$ is the inclusion map.

(A) Since the dense subalgebra $\mathscr{A} \subseteq \mathscr{B}$ is unital, so is $\mathscr{B}$. Thus $\mathscr{A}, \mathscr{B}$ are both unital and hence the primitive ideal spaces $\operatorname{Prim}(\mathscr{A}), \operatorname{Prim}(\mathscr{B})$ compact. 
By Theorem 1, the map $\Phi: Q \in \operatorname{Prim}(\mathscr{B}) \rightarrow Q \cap \mathscr{A} \in \operatorname{Prim}(\mathscr{A})$ is continuous and one to one. Assume $\Phi$ is not surjective. Then $K=\Phi(\operatorname{Prim}(\mathscr{B}))$ is a proper compact subset of $\operatorname{Prim}(\mathscr{A})$ and by the regularity of $\mathscr{A}, \operatorname{ker}(K) \subseteq \mathscr{A}$ contains an element $a \in \mathscr{A}$ with $\rho_{p}(a)>0$. But then $a \in \cap \operatorname{Prim}(\mathscr{B})=$ $\operatorname{rad}(\mathscr{B})$ and hence $\rho_{\mathscr{B}}(a)=0$. But this contradicts $\rho_{\mathscr{B}}(a) \geq \rho_{p}(a)>0$. Thus $\Phi$ must be onto. Now if $\mathscr{A}$ is completely regular and hence $\operatorname{Prim}(\mathscr{A})$ Hausdorff, it follows that $\Phi$ is a homeomorphism.

(B) Let $\pi$ be an irreducible representation of $\mathscr{A}$ on $X$ and $P=\operatorname{ker}(\pi)$. By (A) there exists a primitive ideal $Q \subseteq \mathscr{B}$ such that $P=Q \cap \mathscr{A}$. Choose a continuous irreducible representation $\sigma$ of $\mathscr{B}$ on some Banach space $Y$ such that $Q=\operatorname{ker}(\sigma)$ and set $\sigma_{0}=\sigma_{\mathscr{A}}$. Then $\sigma_{0}$ is an irreducible representation of $\mathscr{A}$ on $Y$ and $\operatorname{ker}\left(\sigma_{0}\right)=\operatorname{ker}(\pi)$. Notice that $X, Y$ are finite dimensional since $\mathscr{A}, \mathscr{B}$ are strong Moore algebras. By Lemma 3 there exists a linear isomorphism $U: X \rightarrow Y$ such that $\pi(a)=U^{-1} \sigma(a) U$ for all $a \in \mathscr{A}$. It is now clear that $b \rightarrow U^{-1} \sigma(b) U$ is an irreducible representation of $\mathscr{B}$ on $X$ that extends $\pi$.

(C) Since the map $Q \in \operatorname{Prim}(\mathscr{B}) \rightarrow Q \cap \mathscr{A} \in \operatorname{Prim}(\mathscr{A})$ is onto, $\operatorname{rad}(\mathscr{B}) \cap$ $\mathscr{A}=\bigcap \operatorname{Prim}(\mathscr{B}) \cap \mathscr{A}=\bigcap \operatorname{Prim}(\mathscr{A})=(0)$, by semisimplicity of $\mathscr{A}$.

(D) Let $a \in \mathscr{A}$ and assume $a \in \operatorname{inv}(\mathscr{B})$. Then $1-a$ is quasi-regular and hence quasi-regular modulo every primitive ideal $Q \subseteq \mathscr{B}$. By Lemma 2 , the map $x+Q \cap \mathscr{A} \in \mathscr{A} /(Q \cap \mathscr{A}) \rightarrow x+Q \in \mathscr{B} / Q$ is an isomorphism. It follows that $(1-a)+Q \cap \mathscr{A}$ is quasi-regular in $\mathscr{A} /(Q \cap \mathscr{A})$. Thus $1-a$ is quasi-regular modulo every primitive ideal in $\mathscr{A}$ and hence $[3,2.2 .9]$ is quasi-regular in $\mathscr{A}$. This means that $a$ is invertible in $\mathscr{A}$.

(E) Follows easily from (C).

(F) Let \|\| be a continuous submultiplicative norm on $\mathscr{A}$ and $\mathscr{B}$ the \|\|$-$ completion of $\mathscr{A}$. Then for every $a \in \mathscr{A}$ we have $\|a\| \geq \lim _{n \uparrow \infty}\left\|a^{n}\right\|^{1 / n}=$ $\rho_{\mathscr{B}}(a)=\rho_{\mathscr{A}}(a)$.

Proof of Theorem 3. This follows from Theorem 2 by adjoining identities if necessary. Recall that the unitization of a completely regular Banach algebra is again completely regular.

Corollary 1. Let $\mathscr{A}$ be a unital, ${ }^{*}$-semisimple Banach ${ }^{*}$-algebra. If $\mathscr{A}$ is a regular strong Moore algebra then $\mathscr{A}$ is symmetric.

Proof. This follows from the fact that the largest $B^{*}$-norm $p$ on $\mathscr{A}$ satisfies $p(a) \geq \rho(a)$ for all $a \in \mathscr{A}$.

As a further application we obtain the following (well-known)

Corollary 2. If $G$ is a regular Moore group, then $G$ is amenable and symmetric. Proof. This follows from the fact that the $B^{*}$-norm $q$ on $L^{1}(G)$ that is induced by the left regular representation of $L^{1}(G)$ on $L^{2}(G)$ satisfies $q(a) \geq \rho(a)$ for all $a \in \mathscr{A}$, and hence coincides with the largest $B^{*}$-norm on $L^{1}(G)$.

Remarks. (1) Regularity of $\mathscr{A}$ is not necessary for the extension result (B) of Theorem 2 to hold. To see this assume that $\mathscr{A}$ is commutative and densely embedded in the Banach algebra $\mathscr{B}$. Then $\mathscr{B}$ is commutative also. Clearly a multiplicative linear functional $\chi$ on $\mathscr{A}$ extends to $\mathscr{B}$ (by continuity) if and only if it satisfies $|\chi(a)| \leq\|a\|$ for all $a \in \mathscr{A}$, where \|\| denotes the complete norm on $\mathscr{B}$. Since $|\chi(a)| \leq \rho_{\mathscr{A}}(a)$, all multiplicative linear functionals will 
extend from $\mathscr{A}$ to $\mathscr{B}$, if the complete norm on $\mathscr{B}$ satisfies $\|a\| \geq \rho_{\mathscr{A}}(a)$ for all $a \in \mathscr{A}$. Now let $\mathscr{A}$ be the algebra of all continuous functions on the unit disc $D \subseteq \mathbb{C}$ that are holomorphic on the disc $D_{0}=\{z \in \mathbb{C}:|z|<1 / 2\}$. It is shown in [6] that every submultiplicative norm \|\| on $\mathscr{A}$ satisfies $\|a\| \geq \rho_{\mathscr{A}}(a)$ for all $a \in \mathscr{A}$. Thus every multiplicative linear functional on $\mathscr{A}$ extends to all Banach algebras $\mathscr{B}$ in which $\mathscr{A}$ is densely (not necessarily continuously) embedded. The primitive ideal space of $\mathscr{A}$ can be identified with the unit disc $D$. Any compact subset of $D_{0}$, which has a cluster point, cannot be annihilated by a nonzero element $f \in \mathscr{A}$.

(2) If the strong Moore property is dropped, then Theorem 2 is no longer true, even if the algebra $\mathscr{A}$ is regular. Recall that regularity of $\mathscr{A}$ requires certain closed two-sided ideals of $\mathscr{A}$ to contain elements of positive permanent radius. Below we give an example of a primitive (and hence semisimple) Banach algebra $\mathscr{A}$ such that every, not necessarily closed, nonzero, two-sided ideal in $\mathscr{A}$ contains an idempotent $e$ (then $\rho_{p}(e) \geq 1$ ) and that violates conclusion $(\mathrm{F})$ of Theorem 2:

Let $H:=l_{2}$ with $|x|_{2}^{2}:=\sum\left|x_{k}\right|^{2}, x=\left(x_{k}\right) \in H$ be separable Hilbert space and $\mathscr{B}(H)$ the associated operator algebra. Now consider a second linear norm ||$_{w}$ on $H:|x|_{w}^{2}:=\sum 4^{-k}\left|x_{k}\right|^{2}$. Note that ||$_{w} \leq||_{2} ;$ let ||$_{2}^{\text {op }},||_{w}^{\text {op }}$ be the associated operator norms and $\mathscr{A}:=\left\{T \in \mathscr{B}(H):|T|_{w}^{\text {op }}<\infty\right\}$ be the algebra of all linear operators on $H$ that are continuous with respect to both norms $\left.\right|_{2}$ and $\left.\right|_{w}$ on $H$. Note that $\mathscr{A}$ is a Banach Algebra under the norm ||$:=\max \left\{||_{2}^{\text {op }},|| \stackrel{\text { op }}{w}\right\}$. Let $\mathscr{F}(H)$ be the ideal of bounded finite rank operators on $H$. The same argument as for the full operator algebra $\mathscr{B}(H)$ shows that every ideal in $\mathscr{A}$ contains the ideal $\mathscr{F}(H) \cap \mathscr{A}$ and hence rank one projections.

Note that $\mathscr{A}$ contains the (unilateral) right shift operator $\sigma: x=\left(x_{k}\right) \rightarrow$ $\left(0, x_{0}, x_{1}, \ldots\right)$. In fact $|\sigma|_{w}^{\mathrm{op}}=\frac{1}{2}$ and $|\sigma|_{2}^{\mathrm{op}}=1$. However, $\sigma$ has spectral radius 1 in the algebra $\mathscr{B} \supseteq \mathscr{A}$, hence $\rho_{\mathscr{A}}(\sigma) \geq 1$. Thus the submultiplicative norm ||$_{w}^{\text {op }}$ on $\mathscr{A}$ satisfies $|\sigma|_{w}^{\text {op }}<\rho_{\mathscr{A}}(\sigma)$.

\section{REFERENCES}

1. G. E. Silov, On the extension of maximal ideals, Dokl. Akad. Nauk SSSR 29 (1940), 83-84.

2. C. E. Rickart, On spectral permanence for certain Banach algebras, Proc. Amer. Math. Soc. 4 (1953), 191-196.

3. C. E. Rickart, General theory of Banach algebras, Krieger, New York, 1960.

4. B. A. Barnes, Ideal and representation theory of the $L^{1}$-algebra of a group with polynomial growth, Colloq. Math. 45 (1981), 301-315.

5. R. D. Mosak, A note on Banach representations of Moore groups, Resultate Math. 5 (1982), 177-183.

6. M. J. Meyer, The spectral extension property and extension of multiplicative linear functionals, Proc. Amer. Math. Soc. 112 (1991), 885-861.

7. I. Kaplanski, Normed algebras, Duke Math. J. 16 (1949), 399-418.

8. H. G. Dales, Automatic continuity: a survey, Bull. London Math. Soc. 10 (1978), 129-183.

9. B. Aupétit, Propriétés spectrales des algèbres de Banach, Lecture Notes in Math., vol. 735, Springer-Verlag, Berlin, 1979.

10. T. W. Palmer, Spectral algebras, subalgebras and pseudonorms, Rocky Mountain J. Math. (to appear). 
11. B. J. Tomiuk and B. Yood, Incomplete normed algebra norms on Banach algebras, Studia Math. 95 (1989), 119-132.

12. F. F. Bonsall and J. Duncan, Complete normed algebras, Springer-Verlag, Berlin, 1973.

13. C. C. Moore, Groups with finite dimensional irreducible representations, Trans. Amer. Math. Soc. 166 (1972), 401-410.

Department of Mathematics and Computer Science, Georgia State University, Atlanta, Georgia 30303 